\title{
Cortical Activation during Cooperative and Competitive Joint Actions in Children With and Without Autism Spectrum Disorder (ASD) - An fNIRS Study
}

\author{
Wan-Chun Su \\ University of Delaware \\ McKenzie Culotta \\ University of Delaware \\ Daisuke Tsuzuki \\ Tokyo Metropolitan University \\ Anjana Bhat ( $\nabla$ abhat@udel.edu ) \\ University of Delaware
}

\section{Research Article}

Keywords: Cooperative and Competitive Joint Actions, Autism Spectrum Disorder (ASD), spatio-temporal action errors

Posted Date: August 20th, 2021

DOI: https://doi.org/10.21203/rs.3.rs-820947/v1

License: (c) (i) This work is licensed under a Creative Commons Attribution 4.0 International License. Read Full License 


\section{Abstract}

Children with Autism Spectrum Disorder (ASD) have social communication and perceptuomotor difficulties that affect their ability to engage in various types of joint actions. In this study, we compared spatio-temporal action errors and fNIRS-related cortical activation between children with and without ASD during a Lincoln log joint action game requiring them to play leader or follower roles, move in synchrony or while taking turns, and move cooperatively or competitively with an adult partner. Children with ASD had greater motor, planning, and spatial errors and took longer to complete the building tasks compared to typically developing (TD) children. Children with ASD had lower superior temporal sulcus (STS) activation during Turn-take and Compete, and greater Inferior Parietal Lobe (IPL) activation during Lead and Turntake compared to TD children. As joint action demands increased, TD children showed greater STS activation during Turntake (vs. Synchrony) and Compete (vs. Cooperate) whereas children with ASD showed greater IPL activation during Lead and Compete (vs. Cooperate). Our findings suggest that children with ASD rely on self-generated action plans (i.e., increased IPL activation) more than relying on their partner's action cues (i.e., reduced STS activation) when performing joint actions.

\section{Introduction}

Autism Spectrum Disorder (ASD) is a prevalent neurodevelopmental disorder affecting 1 in 54 children. ${ }^{1}$ Children with ASD have primary impairments in social communication, including poor social reciprocity, verbal/nonverbal communication skills, as well as the presence of restricted and repetitive behaviors ${ }^{2}$. Apart from the primary symptoms of ASD, they also present with sensorimotor comorbidities, such as movement incoordination and dyspraxia (i.e., difficulties in performing complex motor sequences) ${ }^{3-6}$, as well as poor executive functions, including poor attention shifting, working memory, response inhibition, and mental flexibility ${ }^{7}$. Together, these primary and comorbid impairments contribute to their difficulties in engaging in joint actions.

Joint actions are complex everyday activities that require one to play different roles (a leaders or followers), move with different temporal demands (in-synchrony or when taking turns), and while using same or different goals (cooperativelysimilar goal or competitive-different/opposite goals). These rich and variable contexts embedded within joint actions provide opportunities for motor learning and to build social connections with others ${ }^{8}$. Difficulties in performing joint actions could affect children with ASD's abilities to learn new skills and establish/maintain social relationships ${ }^{5,9}$. Although studies have posited neural mechanisms underlying social interaction difficulties of children with ASD, cortical activation patterns during different types of joint actions are not well understood. In this study, we use functional near infrared spectroscopy (fNIRS), a non-invasive neuroimaging technique that is robust against motion artifacts, to investigate cortical activation patterns during Lincoln Log-based joint action games that require children with and without ASD to play leader/follower roles (Lead vs. Follow), move in synchrony or while taking turns (Lead, Follow vs. Turn-take), and while moving cooperatively or competitively with adult partners (Lead, Follow, and Turn-take vs. Compete).

\section{Roles during Joint Actions - Leader or Follower}

Individuals play different roles during everyday joint actions by being a leader or a follower. For example, musicians follow the cues from the conductor to achieve musical harmony whereas the conductor leads and directs the orchestra. Based on their roles within cooperative actions, individuals apply different movement strategies. Leaders tend to focus on internally driven behaviors, including planning and monitoring of one's own movements, whereas followers tend to perform externally driven behaviors, such as being socially aware and adjusting to the leader's actions ${ }^{10}$. Specifically, with the goal of moving together, it is said that leaders often reduce their movement variability so that their movements are more predictable, while the followers prioritize reducing the timing gaps between their own and the leader's actions ${ }^{10}$. 
Several functional magnetic resonance imaging (fMRI) studies suggest potential neural mechanisms that support the aforementioned behavioral strategies when playing leader and follower roles. Using a mutually adaptive tapping synchrony paradigm, Fairhurst et al. found greater cortical activation in regions that are important for self-initiated movements, including supplementary motor area, premotor cortex, precuneus, and inferior parietal sulcus, in leaders compared to the followers ${ }^{11}$. When engaging in bimanual movement synchrony using haptic inputs, leaders showed more activation over the primary somatosensory, motor, supplementary motor, as well as dorsolateral prefrontal cortices / middle frontal gyrus (MFG), which are important for motor control and motor planning, while followers showed more activation over the temporoparietal junction or superior temporal sulcus (STS), a part of the mentalizing and social networks ${ }^{12}$. Similarly, an fNIRS study found greater activation in temporoparietal and sensorimotor regions when musicians played the second violin part as followers compared to when they played the first violin part as leaders ${ }^{13}$. Taken together, greater cortical activation over the sensorimotor or prefrontal cortices in the leaders may reflect efforts in controlling and planning their own actions, while the greater temporoparietal activation in the followers may reflect their efforts to adapt to partners, to monitor and infer their partner's actions, and to match their own actions to that of their partner's. In the present study, we compared behaviors and cortical activation patterns between Lead and Follow conditions during a Lincoln Log joint action game.

\section{Temporal Components during Joint Actions - Synchrony or Turn- taking}

Besides different roles, movement timing is also critical to achieve movement goals and ensure appropriate social interactions. While interpersonal synchrony is important for many cooperative tasks, such as moving a heavy object together, turn-taking is embedded in many everyday activities, such as playing games on playgrounds and engaging in back-and-forth conversations. Both synchrony and turn-taking require one to monitor the cues from their social partner, anticipate/predict partner's movements, and adjust one's own movements accordingly, therefore, the systems that support perceptuo-motor integration are of particular importance ${ }^{14}$. In contrast to turn-taking, interpersonal synchrony involves moment-to-moment synchronization and effort of online monitoring and adjustments ${ }^{14}$. Turn-taking, on the other hand, requires one to remember their partner's actions, wait for one's turn, and plan one's own actions; therefore, processes involving working memory, inhibition control, and motor planning will be important ${ }^{15}$.

Many neuroimaging studies suggest an important role for the observation-execution matching systems (OEMS), including inferior frontal gyrus (IFG), superior temporal sulcus (STS), and inferior parietal lobe (IPL), in matching movements with observed actions; a critical component in synchronous actions or turn-taking ${ }^{16,17}$. The STS region is reported to be more active during movement imitation compared to passive observation or execution, therefore, is said to represent visuomotor correspondences between one's own and another's actions ${ }^{18}$. The frontoparietal connections are important for multisensory integration and perceptuomotor control during joint actions ${ }^{19}$. Specifically, the IFG region is important for goal understanding and inferring intentions of observed actions while the IPL region is important for predicting and planning the kinematics of goal-directed actions ${ }^{20-22}$. Other important brain regions include the pre- and post-central gyrus (PCG) and the prefrontal cortices/MFG. PCG includes the primary motor and somatosensory cortices that receive/process sensory information and execute actions ${ }^{23}$. The prefrontal regions, mainly, the MFG, are important for executive functions such as motor planning, working memory, cognitive shifting, and inhibition - a set of mental skills that are important during interpersonal synchrony and turn-taking 24 .

Using fNIRS, we have reported greater activation over the IFG, STS, and IPL regions in healthy adults and children during interpersonal synchrony compared to solo conditions during reaching and postural sway tasks ${ }^{25-28}$. Similarly, during turntaking while having conversations or when playing piano duets, healthy adults showed differential frontotemporal activation suggestive of social monitoring ${ }^{29-30}$. Similar findings have been found when examining cortical activation 
during turn-taking interactions. During a table setting task, adults showed greater IPL activation during turn taking with another partner vs. moving solo or when observing their partner's actions ${ }^{31}$. In the current study, we compared behaviors and cortical activation during naturalistic, Lincoln Log-based joint actions involving synchrony (Lead and Follow conditions) and turn-taking (Turn-take condition) in children with and without ASD.

\section{Intentions during Joint Actions - Cooperative or Competitive}

Cooperation and competition are important social behaviors for humans. When engaging in cooperative tasks, social partners work towards a shared goal to improve their group performance ${ }^{32}$. In contrast, during competitive tasks, the competitors focus on individual goals and would either optimize one's own performance or undermine the performance of their competitor ${ }^{33}$. For both cooperative and competitive behaviors, it is important for one to consider/refer to their competitor's intentions ${ }^{34}$. Social regions such as the bilateral temporoparietal junction and the inferior frontal/prefrontal cortices will again become important to monitor partner's behaviors and to understand the goals and intentions of their actions $^{34}$.

Using a computerized pattern-building game, an fMRI study found common activation over the frontoparietal network during cooperative and competitive behaviors, however, greater orbitofrontal activation was found during cooperative, while greater IPL and medial frontal activation was found during competitive behaviors ${ }^{35}$. Similarly, Liu et al. found fNIRSrelated differential activation in the right IFG during competitive and cooperative disc-building games ${ }^{36}$. Using hyperscanning techniques (i.e., simultaneous scanning of partners), the same research group found significant interbrain neural synchronization over right STS during cooperative and competitive conditions, as well as greater right IPL activation during the competitive condition of the disc-building game ${ }^{37}$. These results support differential activation of IFG for intention understanding during both competitive and cooperative behaviors, and competition-specific increases in IPL activation to support planning of self-initiated actions and self-other distinctions. In the present study, we compared behaviors and cortical activation during Lincoln Log-based cooperative (Lead and Follow and Turn-take) and competitive (Compete) conditions in children with and without ASD.

\section{ASD-related Difficulties in Joint Actions}

Children with ASD have poor perceptuomotor control, executive functioning, and intention understanding, that might lead to difficulties in various types of joint actions ${ }^{2-7,9}$. During a joint improvisational mirroring game that required participants to take lead or follow the leader, children with ASD spent less time in synchrony with their partner, especially when they are in the follower role ${ }^{38}$. They also spent less time synchronizing with the tester during rhythmic actions such as joint marching, clapping, postural sway, and pendulum swaying tasks ${ }^{3-6,9}$. These difficulties have been attributed to their poor visuomotor and inter-limb coordination within solo and social contexts ${ }^{5-6,9}$. Children with ASD also showed difficulties during turn-taking tasks ${ }^{39-40}$. During back-and-forth conversations, children with ASD showed longer turntaking gaps and reduced temporal variability, suggesting poor response inhibition/executive functioning ${ }^{39}$. During cooperative/competitive joint actions, children with ASD may have difficulties inferring intentions of others which might affect their joint action performance ${ }^{40}$.

In terms of cortical activation, children with ASD have atypical activation over the regions important for OEMS (including IFG, STS, and IPL), executive functioning (including the prefrontal cortices/MFG), and intention understanding (including temporoparietal junction/STS and the prefrontal cortices) that might reflect their difficulties in performing different types of joint actions ${ }^{17,41,42}$. Most fMRI studies have investigated ASD-related cortical activation that asked participants to imitate/follow others and reported atypical activation over OEMS regions ${ }^{16-17}$. Using fNIRS, our research group has also reported hypoactivation in the IFG and STS regions along with hyperactivation in the IPL region when children with ASD engaged in synchronous reaching or whole-body sway motions while following the lead of an adult partner ${ }^{27-28}$. Although 
activation differences between leading and turn-taking joint actions are not well-studied; studies have found reduced prefrontal activation in individuals with ASD during executive functioning tasks requiring inhibition control and motor planning ${ }^{41}$. Such atypical prefrontal activation might also present in children with ASD during leading and turn-taking because these tasks require significant motor planning and response inhibition. When parent-child dyads of children with and without ASD engaged in cooperative and competitive actions there were no cortical activation differences even if there was reduced behavioral synchrony in children with $A S^{43}$. Given the known ASD-related differences in social monitoring/intention inferring, perceptuo-motor integration, and response inhibition/executive functioning, we hypothesize that children with ASD will show atypical activation over inferior frontal/prefrontal, STS, and IPL regions during cooperative and competitive joint actions.

Using fNIRS, the current study investigated differences in cortical activation in children with and without ASD during a Lincoln log-based joint action game. This game incorporated conditions with different roles (Lead vs. Follow), movement timing (Synchrony-Lead and Follow vs. Turn-take), and with/without shared goals (Cooperate included Lead, Follow, and Turn-take vs. Compete). We hypothesize that children with ASD will show greater spatio-temporal errors during joint action and differences in cortical activation over the OEMS (i.e., IFG, STS, IPL) and prefrontal cortices (MFG) for all joint action conditions. Specifically, they may have motor inhibition, control, and planning-related differences affecting MFG, PCG, and IPL activation or action matching/social monitoring differences affecting STS activation.

\section{Results}

\section{Differences in Behavioral Errors, Time to Completion, and Hand Preference}

Children with ASD had greater motor errors $(p=0.03)$, planning errors $(p=0.01)$, and spatial errors $(p=0.03$, Fig. 1A \& Supplementary Table S1). Children with ASD also took longer to complete joint action tasks compared to TD children ( $p<$ 0.001 , Fig. 1B \& Supplementary Table S1). Hand preferences did not differ between the two groups as indicated by similar proportions of log pickups using right, left, or both hands ( $p>0.05$, Fig. $1 \mathrm{C})$.

\section{Cortical Activation Differences}

A repeated measures ANOVA with within-subject factors of condition, hemisphere, and region of interest (ROI), betweensubjects factor of group, and covariates of motor performance (using Bruininks-Oseretsky Test of Motor Proficiency-2 Manual Dexterity scores or BOT-2 MD), and hand preference (i.e., proportion of right hand movements) revealed 2-way interactions of $\mathrm{ROI} \times$ group $[\mathrm{F}(3.0,357.4)=5.6, p=0.001]$, condition $\times \mathrm{ROI}[\mathrm{F}(9.3,1106.9)=2.1, p=0.027]$, hemisphere $\times \mathrm{ROI}$ $[\mathrm{F}(3.5,420.6)=4.2, p=0.003], 3$-way interactions of condition $\times \mathrm{ROI} \times$ group $[\mathrm{F}(9.3,1106.9)=2.1, p=0.024]$ and a hemisphere $\times \mathrm{ROI} \times$ group $[\mathrm{F}(3.5,420.6)=3.3, p=0.014]$, as well as a 4-way interaction of condition $\times$ hemisphere $\times \mathrm{ROI} \times$ group $[F(9.8,1171.2)=2.8, p=0.002]$. The 4-way interaction did not covary with BOT-2 MD scores or hand preference, therefore, it was further explored using post-hoc t-tests. The visual representation of averaged oxygenated hemoglobin $\left(\mathrm{HbO}_{2}\right)$ concentration during all four conditions in both groups is shown in Fig. 2. The means and standard errors (SE) of $\mathrm{HbO}_{2}$ concentrations are presented in Supplementary Table S2, and the $p$-values and the direction of effects for significant post- hoc findings are presented in Supplementary Table S3.

\section{Group Differences in Cortical Activation}

Children with ASD had lower activation in left and right STS regions during Turn-take ( $p s<0.01$, Fig. $3 \mathrm{C})$ and lower left STS activation during Compete compared to the TD children ( $p=0.001$, Fig. 3D). They also had lower left STS activation ( $p=0.01$ but did not survive FDR correction, Fig. 3B) during Follow compared to the TD children. In contrast, children with 
ASD had greater activation in the left IPL region during Lead and Turn-take compared to the TD children $(p s<0.001$, Figs. $3 \mathrm{~A}$ and $3 \mathrm{C})$.

\section{Hemispheric Differences in Activation}

For both children with and without ASD, the hemispheric differences were found only in the IPL region, however, the condition for hemispheric differences differs between groups. Specifically, greater right than left hemispheric activation (i.e., right lateralization) was found in the TD children during Lead and Turn-take ( $p s<0.001$, Fig. $4 \mathrm{~A}$ ), whereas in the children with ASD, a similar right lateralization pattern was found during Compete $(p<0.001$, Fig. 4B).

\section{Condition-related Differences in Cortical Activation}

For condition-related differences, we conducted post-hoc analyses based on our original questions. For Lead vs. Follow differences, the differences were only present in children with ASD (Figs. 5A and 5B). Children with ASD had greater bilateral IPL and right MFG activation during the Lead compared to the Follow ( $p s<0.001$, Fig. 5B). For Synchrony-Lead \& Follow vs. Turn-take, TD children had greater right STS activation during Turn-take compared to Lead $(p=0.01$, Fig. $5 \mathrm{C})$, while children with ASD had greater left IPL activation during Lead compared to Turn-take $(p<0.01$, Fig. 5D). There was no significant difference between Follow and Turn-take in TD children and children with ASD (no $p$-value survived the FDR corrections, Figs. 5E and 5F). For Cooperate-Lead, Follow, Turn-take vs. Compete, TD children showed greater right STS activation during Compete than Lead and Follow ( $p s<0.01$, Figs. $5 \mathrm{G}$ and $5 \mathrm{I}$ ). In addition, the TD children showed greater left IPL activation during Compete vs. Turn-take $(p=0.001$, Fig. $5 \mathrm{~K})$. Children with ASD had greater left IPL activation during Lead vs. Compete $(p<0.001$; Fig. $5 \mathrm{H})$ and greater right IPL activation during Compete vs. Follow $(p=0.0001$, Fig. $5 \mathrm{~J}$ ). There were no conditional differences between Turn-take and Compete in children with ASD ( $p$-values did not survive FDR corrections, Fig. 5L).

\section{Correlations between Cortical Activation and Behavioral Performance and Vineland Adaptive Behavioral Scales (VABS) scores}

Correlations between cortical activation and behavioral errors did not survive FDR corrections (Supplementary Table S4). In terms of relations with VABS, the adaptive functioning measure TD children showed several positive/negative correlations between cortical activation and the VABS scores during Lead, Follow, and Turn-Take, but not Compete. A higher VABS score indicates better social, communication, or functional performance, hence, a positive correlation implies greater activation being associated with better VABS performance. In the TD children, greater VABS communication scores were associated with greater right IFG and STS activation $(r=0.364$ and $0.381, p<0.01)$, greater VABS social scores were associated with greater left STS activation $(r=0.341, p<0.01)$, and greater VABS daily living scores were associated with lower right IPL activation during Lead $(r=-0.513, p<0.001)$. Similarly, in TD children, greater VABS communication scores were associated with greater left MFG, right IFG, and right PCG activation ( $r=0.318$ to $0.403, p<0.05)$, and greater VABS social scores were associated with greater left STS activation during Follow $(r=0.341, p<0.01)$. For Turn-take in TD children, greater VABS communication scores were associated with greater right IFG activation $(r=0.434, p=0.001)$, and greater VABS daily living scores were associated with greater left IPL and right IFG activation ( $r=0.404$ to $0.456, p s<$ 0.001 ; Table 1). However, greater VABS daily living scores were associated with lower left PCG activation during Turn-take $(r=-0.356, p<0.01)$. 
Table 1

Correlations between cortical activation and the VABS and SRS scores in children with and without ASD.

\begin{tabular}{|c|c|c|c|c|c|c|c|c|c|}
\hline \multirow[t]{3}{*}{ ROls } & & \multicolumn{4}{|l|}{ TD group } & \multicolumn{4}{|l|}{ ASD group } \\
\hline & & & & & SRS & & & & SRS \\
\hline & & Communication & Social & $\begin{array}{l}\text { Daily } \\
\text { Living }\end{array}$ & & Communication & Social & $\begin{array}{l}\text { Daily } \\
\text { Living }\end{array}$ & \\
\hline \multicolumn{10}{|l|}{ Lead } \\
\hline \multirow[t]{5}{*}{ Left } & MFG & 0.042 & 0.184 & -0.161 & 0.028 & 0.222 & 0.041 & -0.132 & $-0.262^{\star}$ \\
\hline & IFG & 0.078 & 0.047 & 0.170 & 0.158 & $0.284^{\star}$ & 0.029 & -0.084 & -0.178 \\
\hline & PCG & 0.088 & 0.156 & -0.038 & 0.114 & -0.166 & -0.135 & $-0.307 *$ & -0.162 \\
\hline & STS & 0.240 & $0.341 * \star$ & 0.162 & 0.076 & 0.248 & 0.225 & 0.006 & $-0.314^{\star}$ \\
\hline & IPL & 0.005 & .036 & -0.165 & $0.284^{*}$ & $-0.282^{\star}$ & $-0.303^{*}$ & -0.203 & 0.255 \\
\hline \multirow[t]{5}{*}{ Right } & MFG & 0.013 & 0.123 & -0.101 & -0.015 & 0.029 & -0.198 & -0.124 & 0.04 \\
\hline & IFG & $0.364^{\star \star}$ & 0.215 & 0.238 & 0.150 & $0.385^{\star \star}$ & 0.177 & 0.206 & $-0.509 * *$ \\
\hline & PCG & -0.079 & 0.028 & -0.241 & -0.015 & $0.359 * *$ & 0.267 & 0.024 & $-0.339 * *$ \\
\hline & STS & $0.381^{\star \star}$ & $0.264^{*}$ & 0.198 & 0.121 & $0.280^{\star}$ & 0.006 & 0.086 & $-0.525^{\star \star}$ \\
\hline & IPL & $-0.272^{\star}$ & -0.091 & $-0.513^{\star \star}$ & $0.317^{*}$ & $0.393^{\star \star}$ & 0.249 & -0.002 & 0.037 \\
\hline \multicolumn{10}{|c|}{ Follow } \\
\hline \multirow[t]{5}{*}{ Left } & MFG & $0.318^{*}$ & $0.310 *$ & 0.155 & -0.104 & 0.261 & 0.008 & -0.133 & -0.102 \\
\hline & IFG & 0.200 & 0.175 & 0.195 & -0.113 & 0.060 & -0.162 & -0.268 & 0.037 \\
\hline & PCG & 0.091 & 0.105 & -0.102 & 0.059 & -0.069 & 0.097 & -0.060 & -0.249 \\
\hline & STS & $0.267^{\star}$ & $0.341^{\star *}$ & 0.116 & -0.087 & 0.203 & 0.024 & -0.221 & -0.144 \\
\hline & IPL & $0.298^{*}$ & 0.192 & 0.150 & 0.198 & $-0.392^{\star \star}$ & -0.121 & -0.222 & $0.383^{* *}$ \\
\hline \multirow[t]{5}{*}{ Right } & MFG & $0.266^{\star}$ & $0.307^{*}$ & 0.048 & -0.122 & 0.056 & -0.099 & -0.157 & 0.236 \\
\hline & IFG & $0.403^{\star \star}$ & $0.266^{*}$ & $0.291^{*}$ & 0.053 & 0.056 & -0.074 & -0.125 & -0.080 \\
\hline & PCG & $0.347^{\star \star}$ & 0.152 & 0.208 & -0.080 & 0.181 & 0.012 & 0.072 & -0.184 \\
\hline & STS & 0.252 & $0.279 *$ & 0.081 & 0.041 & -0.019 & -0.086 & -0.229 & 0.044 \\
\hline & IPL & -0.038 & -0.036 & -0.211 & 0.068 & 0.176 & 0.104 & -0.102 & $0.285^{\star}$ \\
\hline \multicolumn{10}{|c|}{ Turn-Take } \\
\hline \multirow[t]{5}{*}{ Left } & MFG & 0.169 & 0.155 & 0.161 & 0.034 & 0.060 & -0.152 & -0.094 & 0.066 \\
\hline & IFG & 0.101 & 0.021 & 0.037 & 0.113 & 0.111 & -0.034 & -0.079 & -0.048 \\
\hline & PCG & 0.008 & -0.047 & $-0.356^{\star *}$ & 0.189 & -0.156 & $-0.302^{\star}$ & -0.125 & -0.007 \\
\hline & STS & 0.174 & $0.269 *$ & -0.172 & -0.211 & 0.252 & 0.063 & -0.141 & -0.097 \\
\hline & IPL & $0.297^{*}$ & 0.200 & $0.404^{\star *}$ & 0.051 & $-0.296^{\star}$ & 0.071 & 0.064 & -0.039 \\
\hline
\end{tabular}

*indicates $p<0.05$; **indicates $p<0.01$. Bolded and shaded font indicates $p$-values survived for FDR corrections. 


\begin{tabular}{|c|c|c|c|c|c|c|c|c|c|}
\hline \multirow[t]{3}{*}{ ROls } & & \multicolumn{4}{|l|}{ TD group } & \multicolumn{4}{|l|}{ ASD group } \\
\hline & & VABS_ & VABS_ & VABS- & SRS & VABS_ & VABS_ & VABS- & SRS \\
\hline & & Communication & Social & $\begin{array}{l}\text { Daily } \\
\text { Living }\end{array}$ & & Communication & Social & $\begin{array}{l}\text { Daily } \\
\text { Living }\end{array}$ & \\
\hline \multirow[t]{5}{*}{ Right } & MFG & 0.094 & 0.132 & 0.085 & 0.009 & -0.171 & -0.257 & -0.179 & 0.170 \\
\hline & IFG & 0.434 ** & 0.158 & 0.456 ** & 0.063 & 0.231 & 0.013 & 0.151 & $-0.527 *$ \\
\hline & PCG & 0.130 & 0.077 & -0.029 & 0.042 & 0.104 & -0.087 & 0.025 & -0.137 \\
\hline & STS & $0.320 *$ & 0.201 & 0.055 & -0.203 & $0.368^{* \star}$ & 0.160 & -0.003 & -0.166 \\
\hline & IPL & 0.086 & 0.087 & -0.018 & 0.166 & 0.161 & 0.000 & -0.039 & -0.031 \\
\hline \multicolumn{10}{|c|}{ Compete } \\
\hline \multirow[t]{5}{*}{ Left } & MFG & 0.020 & 0.050 & 0.030 & 0.140 & 0.199 & -0.119 & -0.061 & 0.148 \\
\hline & IFG & 0.132 & 0.104 & 0.084 & 0.213 & 0.005 & -0.220 & -0.181 & 0.042 \\
\hline & PCG & 0.106 & 0.126 & $-0.307 *$ & 0.254 & -0.027 & -0.005 & -0.068 & -0.183 \\
\hline & STS & 0.154 & -0.027 & 0.078 & 0.011 & 0.248 & 0.041 & -0.218 & -0.172 \\
\hline & IPL & -0.038 & 0.059 & -0.232 & 0.120 & -0.178 & -0.146 & 0.108 & 0.144 \\
\hline \multirow[t]{5}{*}{ Right } & MFG & 0.014 & 0.085 & 0.121 & 0.082 & 0.048 & -0.092 & -0.004 & 0.040 \\
\hline & IFG & $0.388 * *$ & $0.283^{*}$ & 0.190 & 0.016 & 0.079 & -0.082 & -0.140 & -0.127 \\
\hline & PCG & 0.222 & 0.147 & 0.015 & 0.166 & $0.325^{\star}$ & 0.101 & -0.011 & 0.038 \\
\hline & STS & $0.397 * \star$ & 0.167 & 0.167 & 0.101 & 0.211 & -0.020 & -0.268 & -0.198 \\
\hline & IPL & -0.023 & -0.098 & -0.098 & 0.238 & 0.191 & 0.083 & 0.045 & $0.398^{\star \star \star}$ \\
\hline
\end{tabular}

Children with ASD showed significant correlations between cortical activation and VABS communication scores only for Lead, Follow and Turn-Take, but not Compete. Moreover, greater VABS communication scores were associated with greater right IFG, PCG, and IPL activation during Lead ( $r=0.359$ to $0.393, p s<0.01)$. In addition, greater VABS communication scores were associated with lower left IPL activation during Follow $(r=-0.392, p<0.01)$. and greater right STS activation during Turn-take ( $r=0.368, p<0.01$; Table 1$)$.

\section{Correlation between Cortical activation and Social Responsiveness Scale (SRS) scores}

No significant correlations were found between cortical activation and SRS scores in the TD group (Table 1). Higher SRS scores indicate poorer social performance, hence, a positive correlation implies greater activation being associated with poor social responsiveness. During Lead, children with ASD with poorer social performance had lower right IFG, PCG, and STS activations $(r=-0.339$ to $-0.525, p s<0.01)$. During Follow, children with ASD with poorer social performance had greater left IPL activation $(r=0.383, p<0.01)$. During Turn-Take, children with ASD with poorer social performance had lower right IFG activation $(r=-0.527, p s<0.001)$. Lastly, during Compete, children with ASD with poorer social performance had greater left IPL activation $(r=0.398, p<0.01$, Table 1$)$.

\section{Discussion}


Previous fMRI studies of joint actions have been limited to simple hand movements and unnatural environments. Most studies focused on imitation and synchrony-based cooperative actions but not turn-taking or competition. Using fNIRS and motion tracking systems, we have reported differences in behavioral performance and cortical activation in healthy adults and school-age children with and without ASD during multiple interpersonal synchrony tasks involving reaching/body sway versus solo actions. ${ }^{25-28}$ In this study, we extend our past work to a novel naturalistic, joint action building game using Lincoln Logs in children with and without ASD. We found that children with ASD had greater motor, planning, and spatial errors, and they took longer to complete the tasks compared to the TD children. For group-based activation differences, children with ASD had lower bilateral STS activation during Turn-take, and lower left STS activation during Compete and a similar statistical trend for Follow compared to TD children. In contrast, children with ASD had greater left IPL activation during Lead and Turn-take compared to TD children. For hemispheric differences, TD children had right lateralized IPL activation during Lead and Turn-take, whereas children with ASD had right lateralized IPL activation during Compete. For condition-related differences, TD children had a consistent pattern of greater right STS activation during joint action tasks involving greater social monitoring/intention inferring demands (Turn-take $>$ Lead, Compete > Lead, and Compete > Follow). They also had greater left IPL activation during Compete vs. Turn-take. In contrast, children with ASD had a completely different strategy of greater left and/or right IPL or right MFG activation during Lead vs. Follow, Lead vs. Turn-take, Lead vs. Compete, and Compete vs. Follow. For correlations between the cortical activation and the adaptive functioning measure in both groups, better VABS social, communication, and daily living performance was associated with greater left MFG, right IFG, and bilateral STS activation. The associations between VABS scores and bilateral PCG/IPL activation were not consistent. For correlations between cortical activation and SRS scores, children with ASD's poor social performance was also associated with lower right IFG, PCG, and STS activation and greater left/right IPL activation across multiple joint action conditions.

We found that children with ASD had greater motor, spatial, and planning errors and took longer to complete the tasks compared to their TD peers. Children with ASD have poor social awareness, visuo-motor coordination, and executive functioning skills, which might affect their joint building abilities ${ }^{44-47}$. Poor social monitoring is a fundamental diagnostic impairment and is widely reported in children with ASD. Children with ASD are less likely to follow an adult partner's gaze or gestural bids to observe objects in the environment ${ }^{44,47}$. Toddlers with ASD who were shown $2 \mathrm{D}$ clips of a complex scene involving objects and people pay less attention to interacting adults and paid more attention to the surrounding background ${ }^{48}$. Moreover, dyspraxia (i.e., difficulties performing skilled motor sequences) is often reported in children with ASD with greater spatio-temporal errors and greater time to task completion compared to those without ASD ${ }^{3-6}$. Together, these motor coordination/planning and social impairments could impair joint action performance in children with ASD.

Children with ASD had lower bilateral STS activation during Turn-take and lower left STS activation during Compete and Follow compared to TD children. In contrast, they had greater left IPL activation during Lead and Turn-take than the TD children. Recent studies have reported that followers showed greater STS activation whereas leaders showed greater supplementary motor area and sensorimotor activation during synchronous movement or playing of the violin ${ }^{12-13}$. When planning joint actions, children with ASD may rely more on internal or self-generated plans than being externally driven (i.e., using social information from their partners to plan their actions). For hemispheric differences, TD children had right lateralized IPL activation during Lead and Turn-take conditions whereas children with ASD had a similar right lateralized IPL activation during the Compete condition. Right IPL is important in making self-other distinctions when engaging in synchrony or competition vs. cooperation tasks ${ }^{35,49}$. Less right IPL activation is expected when cooperating as it may involve greater merging of self and other whereas greater right IPL activation is expected when competing with a partne $^{35}$. In the present study, children with ASD also showed a similar pattern of greater right lateralized IPL activation during Compete vs. Cooperate. Interestingly, TD children showed a similar pattern of greater right-lateralized IPL activation in Lead and Turn-take; this may also be attributed to greater deactivation in left IPL across multiple joint action conditions. The left IPL region is considered part of the Default Mode Network (DMN) and is said to deactivate when performing 
externally directed processing (i.e., tasks that are cognitively demanding, goal-directed, or requiring greater social awareness $)^{28,50}$. The DMN is said to be important during social as well as imitation tasks ${ }^{51}$. In fact, being imitated led to greater DMN deactivation, compared to when imitating others suggesting that an individual is perhaps more socially aware of their partner's actions when they are able to regulate the social interaction ${ }^{52}$. Consistent with this finding, TD children showed more deactivation in the left IPL across multiple joint action tasks whereas such left IPL deactivation was rarely seen in children with ASD. Past studies have also reported a lack of DMN deactivation in children with ASD during cognitively demanding tasks $28,53,54$.

For most joint action comparisons, TD children had a consistent pattern of greater right STS activation during joint action tasks involving greater social monitoring/intention inferring demands (Turn-take vs. Lead, Compete vs. Lead, and Compete vs. Follow). They also had reduced deactivation in the left IPL during Compete vs. Turn-take suggesting that they were more internally driven when Competing vs. when Cooperating/Turn-taking. In contrast, children with ASD had greater bilateral IPL and right MFG activation during Lead vs. Follow, greater left IPL activation during Lead vs. Follow, Lead vs. Turn-take, and Lead vs. Compete, and greater right IPL activation during Compete vs. Follow. Taken together, TD children had greater activation in an important social network region (i.e., the STS) suggesting they may be more socially aware during joint action tasks involving greater social monitoring/intention inferring demands (i.e., Follow, Turn-take, and Compete vs. Lead). In addition, they might utilize more internally driven/self-initiated planning when competing as seen by reduced left IPL deactivation.

In contrast, children with ASD seem to use a completely different IPL-based strategy wherein they showed reduced deactivation and greater left IPL activation during Lead compared to other joint action conditions, indicating greater reliance on self-generated action plans. Lastly, they also showed greater right IPL activation during Compete vs. Cooperate. Taken together, children with ASD may engage in better self-other distinctions when Leading and Competing with partners compared to Following and Cooperating with others making Leading and Cooperation valuable learning/intervention contexts to promote greater self vs. other awareness.

For correlations between activation and the adaptive functioning in both groups, better VABS social, communication, and daily living performance was generally associated with greater left MFG, right IFG, and bilateral STS activation. For correlations between cortical activation and SRS scores, children with ASD that had better social performance had higher right IFG, PCG, and STS activation and lower left/right IPL activation across multiple joint action conditions. During joint actions, one must anticipate their partner's actions by observing them, infer their intentions, plan one's own actions, and execute the action plan ${ }^{14}$. The networks formed by MFG, IFG, and STS regions are implicated in each of these processes (i.e., social awareness (STS), intention inferring (IFG), planning actions (MFG and IPL), and action execution (PCG) along with many other important brain regions.

Finally, this is a pilot study involving a relatively small sample size $(N=30)$; yet several significant and meaningful findings were revealed. Despite matching multiple factors across groups, we included children with ASD with a broad range of functioning, which may have increased the variability of our study sample. During naturalistic play, it is difficult to control the time to task completion and standardize the duration of the stimulation period. While we followed consistent probe placement, variation in participant head sizes and probe placement could have led to inconsistency in our spatial registration output.

Our study identified multiple behavioral and fNIRS-based neurobiomarkers during a Lincoln Log-based joint action game across prefrontal, frontal, temporal and parietal cortices. Children with ASD had greater behavioral errors (motor, spatial, and planning) and took greater time to complete tasks. In addition, children with ASD showed reduced STS activation and increased IPL activation as well as a lack of differential activation in the STS region compared to TD children. We also found different patterns of activation in children with ASD compared to TD children suggesting that both groups used different mechanisms to process social-perceptual information for motor planning/execution of joint actions. In the 
future, we will use the aforementioned fNIRS neurobiomarkers to assess changes in cortical activation following a bout of socially embedded motor intervention focused on imitation, synchronization, and cooperation. Overall, fNIRS appears to be a valid and powerful child-friendly tool to examine cortical activation during joint play in both children with and without ASD. From a learning standpoint, clinicians must consider utilizing opportunities for leading and competition to improve social awareness in children with ASD apart from following and cooperation.

\section{Methods}

\section{Participants}

Thirty children with and without ASD (Average \pm SE: ASD: $11.5 \pm 0.8,12$ males, 3 females; TD: $12.2 \pm 0.9,8$ males, 7 females) participated. There were no significant group differences in age or ethnicity. Children were recruited through online postings, phone calls, and fliers sent to ASD advocacy groups, and Simons Powering Autism Research (SPARK) participant research match service. SPARK informs their family database about research studies (https://www.sfari.org/resource/spark/). Before participation, we completed screening interviews with potential participants to obtain their demographic information and to confirm their eligibility. The inclusion criteria for children with ASD were (i) should hold a professionally confirmed ASD diagnosis, supported by school records, an Individualized Education Plan for ASD-related services, or medical/neuropsychological records from a psychiatrist or clinical psychologist using the Autism Diagnostic Observation Schedule; there is a growing trend of using professionally confirmed diagnostic records for ASD cohort studies ${ }^{55}$ and (ii) met criteria for a social communication delay (>12 points) on the Social Communication Questionnaire (SCQ) ${ }^{56}$. Children with ASD were excluded if they had any behavioral/sensory issues that prevented them from completing the test activities. The age-matched TD children were excluded if they had any neurological or developmental disorder/delay or a family history of ASD.

Parents of all children completed the Coren handedness survey to assess hand preferences ${ }^{57}$, the VABS measure to assess adaptive functioning, ${ }^{58}$ and SRS to assess social responsiveness impairment ${ }^{59}$. Additionally, we administered the BOT-2 MD to assess fine motor skills ${ }^{60}$. Compared to TD children, children with ASD had significantly lower VABS, BOT-2 MD scores, and greater SRS total scores indicating impaired adaptive functioning, manual dexterity performance, and social responsiveness (Table 2). All study procedures were carried out in accordance with the Declaration of Helsinki. All informed consent and assent forms as well as all study procedures were approved by the University of Delaware Institutional Review Board (UD IRB, Study Approval \#: 930721). Prior to study participation, written informed consent was obtained from parents who gave approval for their child's study participation as their legal guardians and written and verbal assent was obtained from the children. Written parental permission and experimenter informed consent has been taken to use pictures for this publication. 
Table 2

Demographic information and questionnaires data for ASD and TD groups.

\begin{tabular}{|c|c|c|}
\hline \multirow[t]{2}{*}{ Characteristics } & $\begin{array}{l}\text { TD group } \\
(n=15)\end{array}$ & $\begin{array}{l}\text { ASD group } \\
(n=15)\end{array}$ \\
\hline & Mean \pm SE & Mean \pm SE \\
\hline Age & $12.2 \pm 0.9$ & $11.5 \pm 0.8$ \\
\hline Sex & $8 \mathrm{M}, 7 \mathrm{~F}$ & $12 \mathrm{M}, 3 \mathrm{~F}$ \\
\hline Ethnicity & $12 \mathrm{C}, 2 \mathrm{~A}, 1 \mathrm{AA}$ & $11 \mathrm{C}, 2 \mathrm{~A}, 2 \mathrm{AA}$ \\
\hline Handedness & 14R, 1L & $13 \mathrm{R}, 2 \mathrm{~L}$ \\
\hline VABS (\%) & $67.7 \pm 6.0 *$ & $4.2 \pm 1.3$ \\
\hline Composite Score & $65.8 \pm 5.7^{\star}$ & $4.6 \pm 1.3$ \\
\hline Communication & $77.4 \pm 4.1^{\star}$ & $4.8 \pm 1.8$ \\
\hline Socialization & $74.8 \pm 5.8^{*}$ & $8.7 \pm 3.4$ \\
\hline \multicolumn{3}{|l|}{ Daily Living } \\
\hline BOT MD Raw Scores & $26.3 \pm 1.5^{\star}$ & $21.4 \pm 1.9$ \\
\hline SRS Total score & $22.5 \pm 4.2^{\star}$ & $111.1 \pm 7.1$ \\
\hline \multicolumn{3}{|c|}{$\begin{array}{l}\text { SE = Standard error, VABS = Vineland Adaptive Behavioral Scale, } 2 \text { nd edition; BOT MD = Manual Dexterity subtest of } \\
\text { the Bruininks-Oseretsky Test of Motor Proficiency, } 2 \text { nd edition; } S R S=\text { Social Responsiveness Scale, } 2 \text { nd Edition; } M= \\
\text { Male, } F=\text { Female, } C=\text { Caucasian, } A=A \text { sian, } A A=A \text { African-American, } R=\text { Right-handed, } L=\text { Left-handed, *indicates a } \\
\text { significant difference between groups (i.e., } p \text {-value }<0.05 \text { ). }\end{array}$} \\
\hline
\end{tabular}

\section{Experimental Procedures}

Each child sat at a table across from an adult tester and was fitted with a $3 \times 11 \mathrm{fNIRS}$ probe set (Fig. 6A). A container of Lincoln logs consisting of four plain brown logs and four multi-colored (green, yellow, purple, blue) supporting logs was placed on the table and a cue card was placed facing one or both participants. We used a randomized block design comprised of 16 trials in 4 blocks and 4 conditions (Lead, Follow, Turn-take, Compete; Fig. 6B). In the Lead condition, the child built the configuration according to the cue card (shown to the child only) while making sure that the follower/adult tester followed their actions simultaneously. In the Follow condition, the child mimicked the building actions and moved synchronously with the tester who was the only one shown the cue card. In the Turn-take condition, the cue card was visible to both partners. They took turns to make the next move and built the log configurations together. In the Compete condition, non-identical cue cards were given to both, tester and child, and they were asked to quickly and independently build the structure shown on their cue card. Each trial included a 10-second pre-stimulation, 15-second stimulation, and a 15-second post-stimulation period (Fig. 6B). During the pre-and post-baseline periods, participants were asked to observe a crosshair on the wall.

\section{Data Collection}

The Hitachi ETG-4000 system was used to capture the hemodynamic changes during the joint action tasks (Hitachi Medical Systems, Tokyo, Japan, Sampling Rate: $10 \mathrm{~Hz}$ ). A cap embedded with a 3×11 probe set (including 17 infrared emitters and 16 receivers) was positioned over frontal, temporal and parietal regions of the brain (See Supplementary 
Figures S1A and S1B). The midline of the probe set was aligned with the nasion and the lower border of the probe set was aligned just above the eyebrow and the ears. The adjacent pairs of probes, located $3 \mathrm{~cm}$ apart, acted as emitters and receivers for two wavelengths of light (695 and $830 \mathrm{~nm}$ ). Light waves travel from the emitter through the skull, creating a banana-shaped arc reaching the capillary bed of the cortical tissue of the brain. Some of the light waves are absorbed/scattered while the remaining waves are transmitted back to the receivers. Using the Modified Beer-Lambert law, change in light attenuation is used to determine changes in the concentration of $\mathrm{HbO}_{2}$ and deoxygenated hemoglobin $(\mathrm{HHb})$ at the midpoint of two probes, also termed a channel. When a certain cortical region is more active, there will be an increase of metabolic demand/oxygen consumption and blood flow to the capillary bed supplying that cortical region, which in turn leads to an increase in $\mathrm{HbO}_{2}$, and a slight decrease in $\mathrm{HHb}^{61}$. E-prime 2.0 software was used to trigger the ETG system and mark the baseline and stimulation periods. The session was videotaped using a camcorder that was synchronized with the ETG-4000 system.

\section{Spatial Registration Approach}

We recorded the 3D location of standard cranial landmarks (nasion, inion, right/left ear) and each fNIRS probe with respect to a reference coordinate system using a Polhemus motion analysis system. Using the anchor-based spatial registration method developed by our co-author, Tsuzuki, the 3D spatial location of each channel was transferred to the Montreal Neurological Institute's coordinate system ${ }^{62}$. The structural information from a database of 17 adults was then used to provide estimates of channel positions in a standardized 3D brain atlas and the LONI Probabilistic Brain Atlas was used to label estimated channel locations based on MRI scans of 40 healthy adults ${ }^{62-64}$. A channel was included if $55 \%$ or more of the channel area (i.e., each channel was modeled as the centroid of sphere) was within a given ROI and was excluded if it was not. A channel was also excluded if its homologue belonged to another ROI. Based on these rules, we assigned 38 out of 52 channels to five ROls in each hemisphere (See Supplementary Figures S1C and S1D and Table S5) as follows: (i) MFG (right: 3,4,14,15,25,36; left: 7,8,17,18,28,38); (ii) IFG (right: 24,34,35,45; left: 29,39,40,50); (iii) PCG (right: 2,13,23; left: 9,19,30); (iv) STS (right: 32,33,43,44; left: 41,42,51,52); (v) IPL (right: 1,11; left: 10,21).

\section{Data Processing}

We have developed custom MATLAB codes that incorporate functions from open-source software such as HOMER-2 and Hitachi POTATo to process the fNIRS data output ${ }^{65-67}$. The processing steps include: (i) band-pass filtering of the signal between 0.01 and $0.5 \mathrm{~Hz}$ to remove high-/low-frequency noise, (ii) wavelet method to remove movement artifacts, (iii) General Linear Modeling to estimate the hemodynamic response, (iv) correction for baseline drifts by subtracting the linear trend between the pre- and post-baselines from values in the stimulation period, and (v) averaging the $\mathrm{HbO}_{2}$ values during stimulation period for each trial, along with visualization of the processed data at each step ${ }^{65-67}$. We report $\mathrm{HbO}_{2}$ data only, as it has a greater signal to noise ratio than the $\mathrm{HHb}$ data and is more often reported in the literature ${ }^{67}$. The reader is referred to our earlier publications for additional details on fNIRS methodology ${ }^{25-28}$.

\section{Behavioral Coding}

A trained student researcher scored the behavioral performance of the children during task completion. Each session was scored for three error types: (i) Motor error: the child dropped a log before placing in the container or knocked over the container; (ii) Planning error: the child hesitated, and then changed placement location; and (iii) Spatial error: the log was placed incorrectly based on color or location. Furthermore, we coded hand preferences by scoring how the child picked up 
each log using their left, right, or both hands. Lastly, we coded the time in seconds to complete each building configuration.

\section{Statistical Analyses}

To assess group differences in frequency of behavioral errors of each type, we conducted non-parametric, Mann-Whitney$\mathrm{U}$ tests. For group differences in time to completion and hand preference (i.e., proportion of right, left or both hand actions) we conducted independent t-tests for each action type. For cortical activation, we conducted a repeated measure ANOVA using within-group factors of condition (Lead, Follow, Compete, Turn-Take), region (MFG, PCG, IFG, STS, IPL), hemisphere (Left, Right), a between-group factor of group (ASD, TD) with BOT-2 MD score and hand preference as covariates. When our data violated Mauchly's test of sphericity, we applied Greenhouse-Geisser corrections. Lastly, Pearson's correlations were used to correlate cortical activation and behavioral performance (both groups), VABS (both groups) and SRS performance (ASD only). To control for multiple comparisons for post-hoc analyses and correlation runs, the Benjamini-Hochberg False Discovery Rate (FDR) method was used to adjust the statistically significant cut-off ${ }^{68}$. Specifically, the unadjusted $p$-values were rank ordered from low to high and the statistical significance was declared if the unadjusted $p$-value was less than the $p$-value threshold which was determined by multiplying 0.05 with the ratio of the unadjusted $p$-value rank to the total number of comparisons ( $p$-threshold for $\mathrm{i}^{\text {th }}$ comparison $=0.05 \times \mathrm{i} / \mathrm{n}$; where $\mathrm{n}=$ number of comparisons).

\section{Declarations}

\section{Data Availability Statement}

The datasets generated during and/or analyzed during the current study are available from the corresponding author on reasonable request.

\section{Acknowledgments}

We are grateful to the SPARK children and families who participated in this study. We also thank the SPARK clinical sites and SPARK research participant match service staff for their help with participant recruitment. Researchers may contact SPARK study research participant match service here: https://wp-qa.sparkforautism.org/spark-research-match/ This work was supported by the National Institutes of Health through a shared instrumentation grant awarded to the University of Delaware (Grant \#: 1S100D021534-01, PI: Bhat) and pilot award funding through an Institutional Development Award (IDeA) from the National Institute of General Medical Sciences of the National Institutes of Health (U54-GM104941, P20 GM103446) and funding from the Dana Foundation through a Clinical Neuroscience Award (PI: Bhat). The last author's effort in writing and editing this manuscript was also supported by the NIH R01 grant \#: 1R01MH125823-01. The content of this manuscript has been published before as part of the Master's thesis of McKenzie Culotta (2019).

\section{Author Contributions}

M.C., W.S., and A.B. contributed to conception and design of the study, data collection, and organized the database. A.B. and M.C. performed the statistical analyses and wrote the first draft of the manuscript. W.S. and D.T. wrote sections of the manuscript. All authors contributed to manuscript revision, read, and approved the submitted version.

\section{Additional Contributions}


The funders had no role in study design, data collection and analysis, decision to publish, or preparation of the manuscript. All the authors declare that they have no conflicts of interest.

\section{Competing Interests}

The funders had no role in study design, data collection and analysis, decision to publish, or preparation of the manuscript. All the authors declare that they have no conflicts of interest.

\section{References}

1. Maenner, M. J. et al. Prevalence of autism spectrum disorder among children aged 8 years - autism and developmental disabilities Monitoring network, 11 sites, united states, 2016. MMWR Surveill. Summ, 69 (4), 1-12 (2020).

2. American Psychiatric Association. Diagnostic and Statistical Manual of Mental Disorders 5th edn (American Psychiatric Publishing, 2013).

3. Bhat, A. N. Is motor impairment in autism spectrum disorder distinct from developmental coordination disorder? A report from the SPARK study. Phys. Ther, 100 (4), 633-644 (2020).

4. Bhat, A. N. Motor impairment increases in children with autism spectrum disorder as a function of social communication, cognitive and functional impairment, repetitive behavior severity, and comorbid diagnoses: a SPARK study report. Autism Res, 14 (1), 202-219 (2021).

5. Kaur, M., Srinivasan, S. M. \& Bhat, A. N. Comparing motor performance, praxis, coordination, and interpersonal synchrony between children with and without autism spectrum disorder (ASD). Res. Dev. Disabil, 72, 79-95 (2018).

6. Kaur, M., Gifford, T., Marsh, K. \& Bhat, A. The effects of robot-child interactions on bilateral coordination skills of typically developing children and one child with autism between 4 to 7 years of age. Journal of Motor Learning and Development, 1 (2), 31-37 (2013).

7. Freeman, L. M., Lock, J., Rotheram-Fuller, E. \& Mandell, D. Brief report: examining executive and social functioning in elementary aged children with autism. J. Autism Dev. Disord, 47, 1890-1895 (2017).

8. Vicaria, I. \& Dickens, L. Meta-analyses of intra and inter-personal coordination. J. Nonverbal Behav, 40, 335-361 (2016).

9. Fitzpatrick, P. et al. Social motor synchronization: insights for understanding social behavior in autism. J. Autism Dev. Disord, 47, 2092-2107 (2017).

10. Bolt, N. K. \& Loehr, J. D. The motor-related brain activity that supports joint action: A review. Acta Psychol, 212, 103218 (2021).

11. Fairhurst, M. T., Janata, P. \& Keller, P. E. Leading the follower: an fMRI investigation of dynamic cooperativity and leader-follower strategies in synchronization with an adaptive virtual partner. Neurolmage, 84, 688-697 (2014).

12. Chauvigné, L. \& Brown, S. Role-specific brain activations in leaders and followers during joint action. Front. Hum. Neurosci, 12, 401 (2018).

13. Vanzella, P. et al. fNIRS responses in professional violinists while playing duets: evidence for distinct leader and follower roles at the brain level. Front. Psychol, 10, 164 (2019).

14. Vesper, C. et al. Joint action: mental representations, shared information and general mechanisms for coordinating with others. Front. Psychol, 7, 2039 (2017).

15. Meyer, M., Bekkering, H., Haartsen, R., Stapel, J. C. \& Hunnius, S. The role of action prediction and inhibitory control for joint action coordination in toddlers. Exp. Child Psychol, 139, 203-220 (2015).

16. Iacoboni, M. Neurobiology of imitation. Curr. Opin. Neurobiol, 19, 661-665 (2009). 
17. Yang, J. \& Hofmann, J. Action observation and imitation in autism spectrum disorders: an ALE meta-analysis of fMRI studies. Brain Imaging Behav, 10, 960-969 (2015).

18. Molenberghs, P., Brander, C., Mattingley, J. B. \& Cunnington, R. The role of the superior temporal sulcus and the mirror neuron system in imitation. Hum. Brain Mapp, 31 (9), 1316-1326 (2010).

19. Limanowski, J. \& Blankenburg, F. Fronto-parietal brain responses to visuotactile congruence in an anatomical reference frame. Front. Hum. Neurosci, 12, 84 (2018).

20. Johnson-Frey, S. H. et al. Actions or hand-object interactions? Human inferior frontal cortex and action observation. Neuron, 39 (6), 1053-1058 (2003).

21. Koski, L. et al. Modulation of motor and premotor activity during imitation of target-directed actions. Cereb. Cortex, 12 (8), 847-855 (2002).

22. Fontana, A. P. et al. Role of the parietal cortex in predicting incoming actions. Neurolmage, 59 (1), $556-564$ (2012).

23. Vrticka, P. et al. Neural substrates of social emotional regulation on imitation and expressive suppression to dynamic facial signals. Front. Psychol, 4 (95), 1-10 (2013).

24. Del Casale, A. et al. Executive functions in obsessive-compulsive disorder: An activation likelihood estimate metaanalysis of fMRI studies. World J. Biol. Psychiatry, 17 (5), 378-393 (2016).

25. Bhat, A. N. et al. Cortical activation during action observation, action execution, and interpersonal synchrony in adults: a functional near-infrared spectroscopy (fNIRS) study. Front Hum Neurosci, 11, 431 (2017).

26. Su, W. C. et al. Developmental differences in cortical activation during action observation, action execution, and interpersonal synchrony in adults: an fNIRS study. Front. Hum. Neurosci, 14, 57 (2020a).

27. Su, W. C. et al. Differences in cortical activation patterns during action observation, action execution, and interpersonal synchrony between children with or without autism spectrum disorder (ASD): An fNIRS pilot study. PLoS one, 15 (10), e0240301 (2020b).

28. Su, W. C., Culotta, M., Tsuzuki, D. \& Bhat, A. Movement kinematics and cortical activation in children with and without autism spectrum disorder during sway synchrony tasks: an fNIRS study. Sci. Rep, 11 (1), 15035 (2021).

29. Huberth, M. et al. Performance monitoring of self and other in a turn-taking piano duet: A dual-EEG study. Soc. Neurosci, 14 (4), 449-461 (2019).

30. Foti, D. \& Roberts, F. The neural dynamics of speech perception: Dissociable networks for processing linguistic content and monitoring speaker turn-taking. Brain. Lang, 157-158, 63-71 (2016).

31. Egetemeir, J., Stenneken, P., Koehler, S., Fallgatter, A. J. \& Herrmann, M. J. Exploring the neural basis of real-life joint action: measuring brain activation during joint table setting with functional near-infrared spectroscopy. Front. Hum. Neurosci, 5, 95 (2011).

32. Lee, M., Ahn, H. S., Kwon, S. K. \& Kim, S. I. Cooperative and competitive contextual effects on social cognitive and empathic neural responses. Front. Hum. Neurosci, 12, 218 (2018).

33. Murayama, K. \& Elliot, A. J. The competition-performance relation: a meta-analytic review and test of the opposing processes model of competition and performance. Psychol. Bull, 138 (6), 1035-1070 (2012).

34. Tsoi, L., Dungan, J., Waytz, A. \& Young, L. Distinct neural patterns of social cognition for cooperation versus competition. Neurolmage, 137, 86-96 (2016).

35. Decety, J., Jackson, P. L., Sommerville, J. A., Chaminade, T. \& Meltzoff, A. N. The neural bases of cooperation and competition: an fMRI investigation. Neurolmage, 23 (2), 744-751 (2004).

36. Liu, T., Saito, H. \& Oi, M. Role of the right inferior frontal gyrus in turn-based cooperation and competition: A nearinfrared spectroscopy study. Brain Cogn, 99, 17-23 (2015).

37. Liu, T., Saito, G., Lin, C. \& Saito, H. Inter-brain network underlying turn-based cooperation and competition: A hyperscanning study using near-infrared spectroscopy. Sci. Rep, 7 (1), 8684 (2017). 
38. Brezis, R. S. et al. Patterns of joint improvisation in adults with autism spectrum disorder. Front. Psychol, 8, 1790 (2017).

39. Ochi, K. et al. Quantification of speech and synchrony in the conversation of adults with autism spectrum disorder. PLoS one, 14 (12), e0225377 (2019).

40. Cerullo, S., Fulceri, F., Muratori, F. \& Contaldo, A. Acting with shared intentions: A systematic review on joint action coordination in autism spectrum disorder. Brain Cogn, 149, 105693 (2021).

41. Uratani, M. et al. Reduced prefrontal hemodynamic response in pediatric autism spectrum disorder measured with near-infrared spectroscopy. Child Adolesc. Psychiatry Ment. Health, 13, 29 (2019).

42. Todorova, G. K., Hatton, R. \& Pollick, F. E. Biological motion perception in autism spectrum disorder: a meta-analysis. Mol. Autism, 10, 49 (2019).

43. Kruppa, J. A. et al. Brain and motor synchrony in children and adolescents with ASD-A fNIRS hyperscanning study. Soc. Cogn. Affect Neurosci, 16 (1-2), 103-116 (2021).

44. Leekam, S. R., Lopez, B. \& Moore, C. Attention and joint attention in preschool children with autism. Dev Psychol, 38, 261-273 (2000).

45. Robinson, S., Goddard, L., Dritschel, B., Wisley, M. \& Howlin, P. Executive functions in children with autism spectrum disorders. Brain Cogn, 71, 362-368 (2009).

46. Sachse, M. et al. Executive and visuo-motor function in adolescents and adults with autism spectrum disorder. J. Autism Dev. Disord, 43, 1222-1235 (2013).

47. Leekam, S. R. \& Ramsden, C. A. Dyadic orienting and joint attention in preschool children with Autism. J. Autism Dev. Disord, 36, 185-197 (2006).

48. Shic, F., Bradshaw, J., Klin, A., Scassellati, B. \& Chawarska, K. Limited activity monitoring in toddlers with autism spectrum disorder. Brain Res, 1380, 246-254 (2011).

49. Ohata, R. et al. Sense of agency beyond sensorimotor process: decoding self-other action attribution in the human brain. Cereb. Cortex, 30 (7), 4076-4091 (2020).

50. Greicius, M. D., Krasnow, B., Reiss, A. L. \& Menon, V. Functional connectivity in the resting brain: a network analysis of the default mode hypothesis. Proc. Natl. Acad. Sci. U. S. A. 100(1), 253-258(2003).

51. Schilbach, L., Eickhoff, S. B., Rotarska-Jagiela, A., Fink, G. R. \& Vogeley, K. Minds at rest? Social cognition as the default mode of cognizing and its putative relationship to the "default system" of the brain. Conscious Cogn, 17 (2), 457-467 (2008).

52. Guionnet, S. et al. Reciprocal imitation: toward a neural basis of social interaction. Cereb. Cortex, 22 (4), 971-978 (2012).

53. Spencer, M. D. et al. Failure to deactivate the default mode network indicates a possible endophenotype of autism. Mol. Autism, 3 (1), 15 (2012).

54. Chien, H. Y., Gau, S. S. \& Isaac Tseng, W. Y. Deficient visuospatial working memory functions and neural correlates of the default mode network in adolescents with autism spectrum disorder. Autism Res, 9 (10), 1058-1072 (2016).

55. Lord, C. et al. Autism diagnostic observation schedule, second edition (ADOS-2) manual (Part 1): Modules 1-4 (Western Psychological Services, Torrance, CA, 2012).

56. Daniels, A. M. et al. Verification of parent-report of child autism spectrum disorder diagnosis to a web-based autism registry. J. Autism Dev. Disord, 42 (2), 257-265 (2012).

57. Coren, S. H. \& Questionnaire The Left-Hander Syndrome: The Causes and Consequences of Left-Handedness (NY Free Press, New York, 1992).

58. Volkmar, F. R. et al. Social deficits in autism: an operational approach using the Vineland Adaptive Behavior Scales. J. Am. Acad. Child Adolesc. Psychiatry, 26 (2), 156-161 (1987).

Page $17 / 22$ 
59. Constantino, J. N. \& Gruber, C. P. Social Responsiveness Scale (SRS) (Western Psychological Services, Los Angeles, 2005).

60. Bruininks, R. \& Bruininks, B. Bruininks-Oseretsky Test of Motor Proficiency: Examiner's Manual (Pearson's Assessments, Minneapolis, MN, 2005).

61. Lloyd-Fox, S., Blasi, A. \& Elwell, C. E. Illuminating the developing brain: The past, present and future of functional near infrared spectroscopy. Neurosci. Biobehav. Rev, 34, 269-284 (2010).

62. Okamoto, M. et al. Three-dimensional probabilistic anatomical cranio-cerebral correlation via the international $10-20$ system oriented for transcranial functional brain mapping., 21, 99-111 (2004).

63. Tsuzuki, D. et al. Stable and convenient spatial registration of stand-alone NIRS data through anchor-based probabilistic registration. Neurosci. Res, 72, 163-171 (2012).

64. Shattuck, D. W. et al. Construction of a 3D probabilistic atlas of human cortical structures., 39, 1064-1080 (2010).

65. Huppert, T. J., Diamond, S. G., Franceschini, M. A. \& Boas, D. A. HomER: a review of time-series analysis methods for near-infrared spectroscopy of the brain. Appl. Opt, 48 (10), 280-298 (2009).

66. Sutoko, S. et al. Tutorial on platform for optical topography analysis tools. Neurophotonics, 3, 010801 (2016).

67. Sato, H. et al. Intersubject variability of near-infrared spectroscopy signals during sensorimotor cortex activation. J. Biomed. Opt, 10, 44001 (2005).

68. Singh, A. \& Dan, I. Exploring the false discovery rate in multichannel NIRS., 33, 542-549 (2006).

\section{Figures}
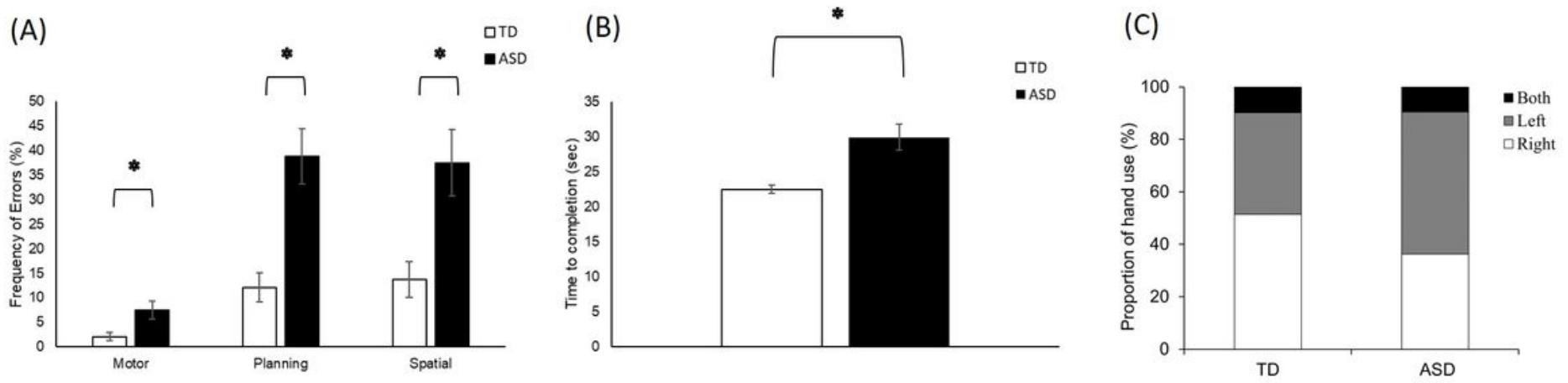

\section{Figure 1}

Behavioral errors (A), Time to completion (B), and Hand preference (C) in children with ASD and TD children. *indicates significant differences between ASD and TD groups. 

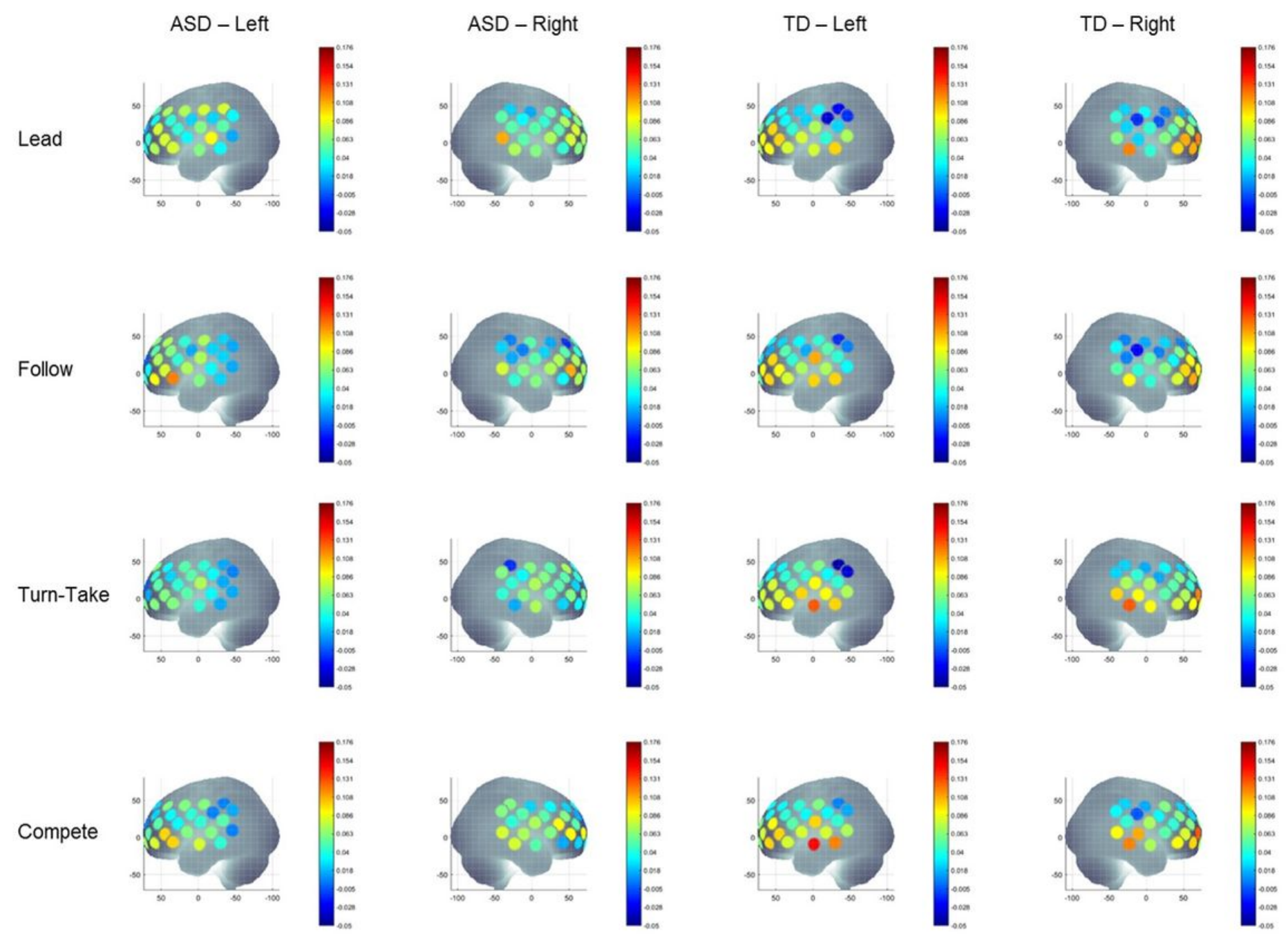

\section{Figure 2}

A visual representation of averaged $\mathrm{HbO} 2$ concentration during Lead, Follow, Turn-take, and Compete conditions in children with ASD and TD children. $\mathrm{HbO} 2$ values on $\mathrm{Y}$-axis range from -0.05 indicated by blue to 0.176 indicated by red. TD children showed greater STS-related channel activation during Compete and Turn-take conditions that is seen in children with ASD. In contrast, children with ASD had greater activation in IPL channels during the Lead, Turn-Take, and Compete. 
(A) Lead

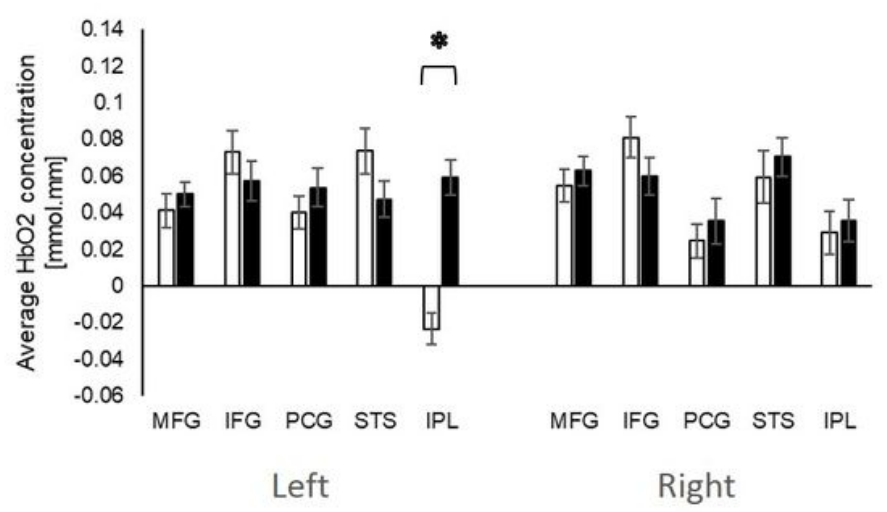

(C) Turn-Take

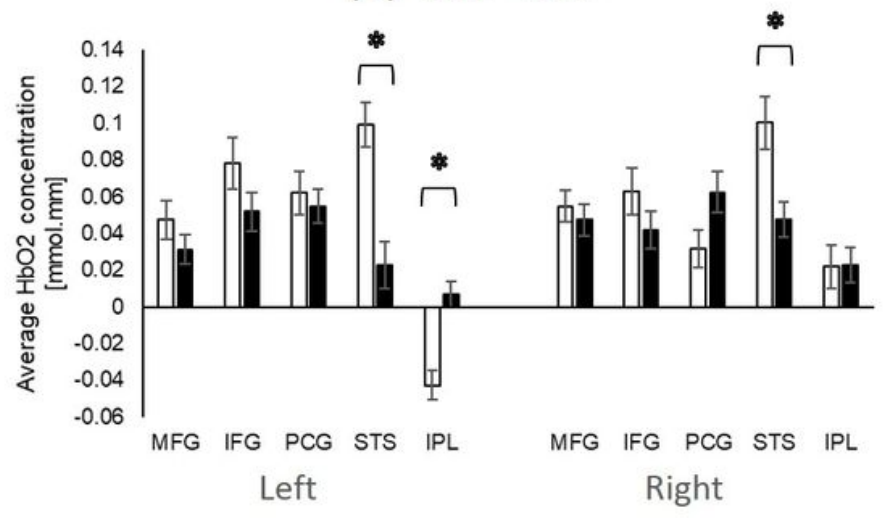

(B) Follow

口TD

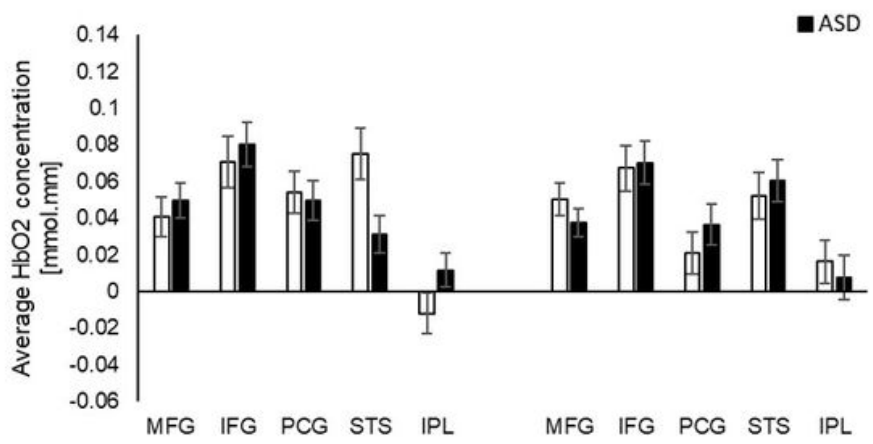

Left

Right

(D) Compete

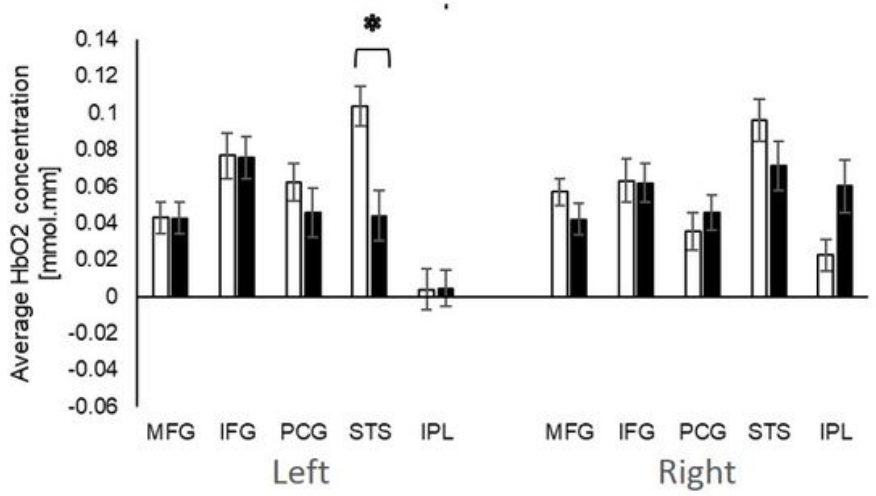

\section{Figure 3}

Group differences in cortical activation for Lead (A), Follow (B), Turn-Take (C), and Compete (D). *indicates significant differences between the ASD and TD groups.

(A) TD

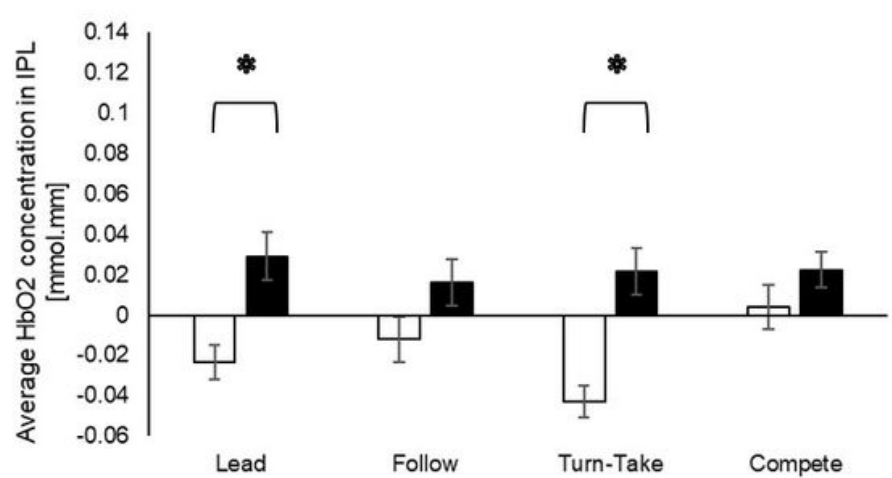

(B) ASD

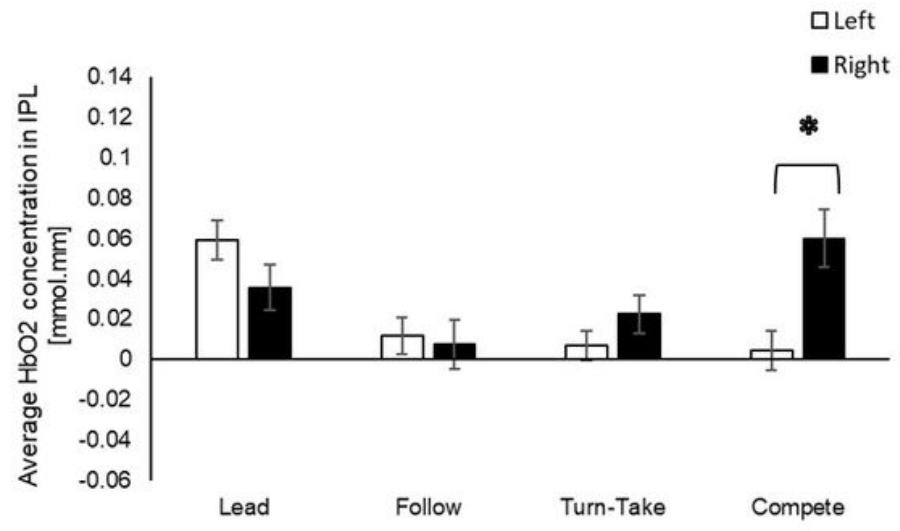

\section{Figure 4}

Hemispheric differences between TD children (A) and children with ASD (B). *indicates significant differences between the ASD and TD groups. 


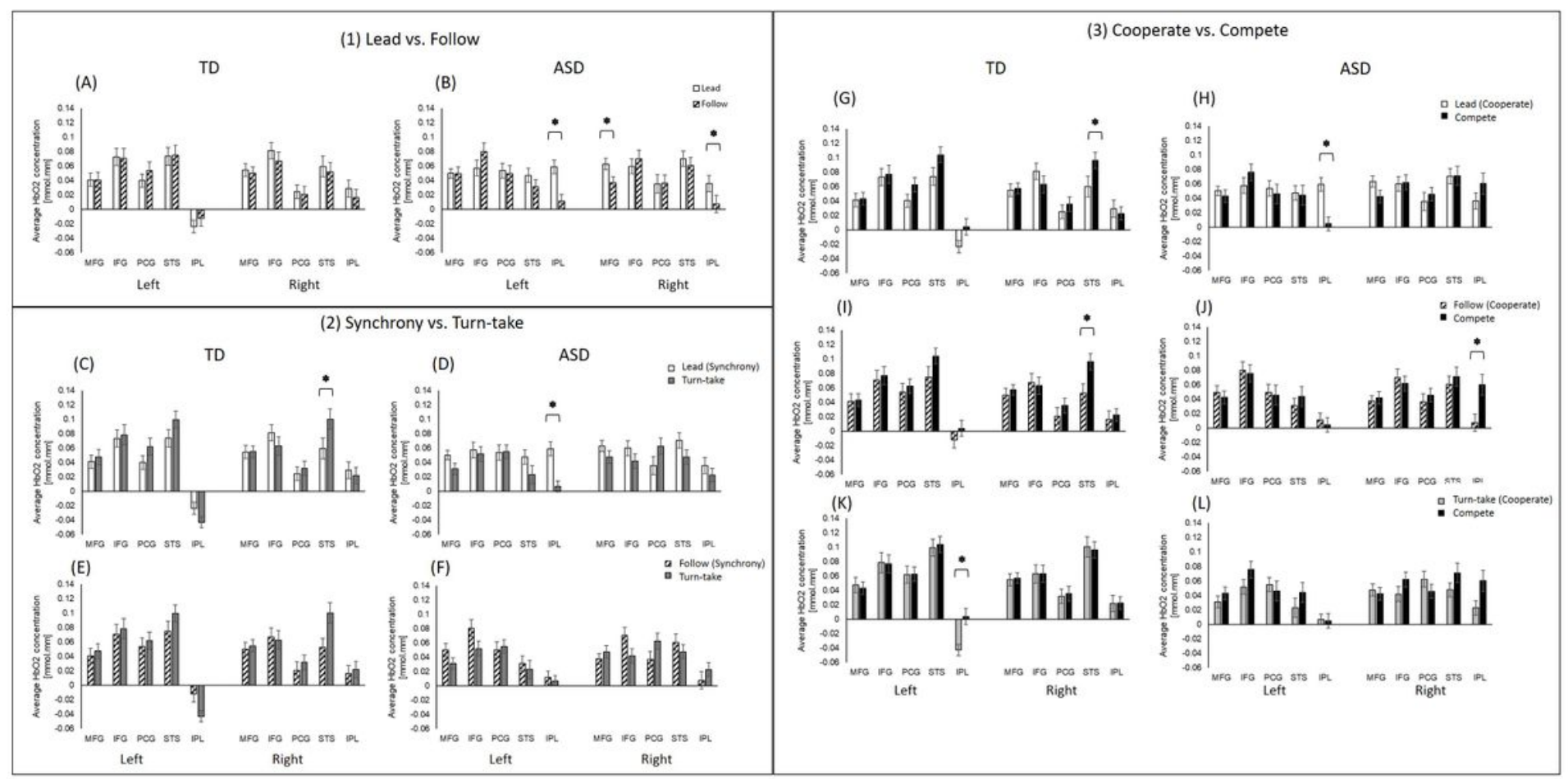

Figure 5

Condition-related differences in cortical activation for TD children and children with ASD for: (1) Lead vs. Follow in TD children (A) and children with ASD (B); (2) Synchrony (Lead and Follow) vs. Turn-take i.e., Lead vs. Turn-take in TD children (C) and children with ASD (D), Follow vs. Turn-take in TD children (E) and children with ASD (F); (3) Cooperative (Lead, Follow, Turn-take) vs. Compete i.e., Lead vs. Compete in TD children (G) and children with ASD (H), Follow vs. Compete in TD children (I) and children with ASD (J), Turn-take vs. Compete in TD children (K) and children with ASD (L). *indicates significant differences between the ASD and TD groups. 


\section{A. Conditions}
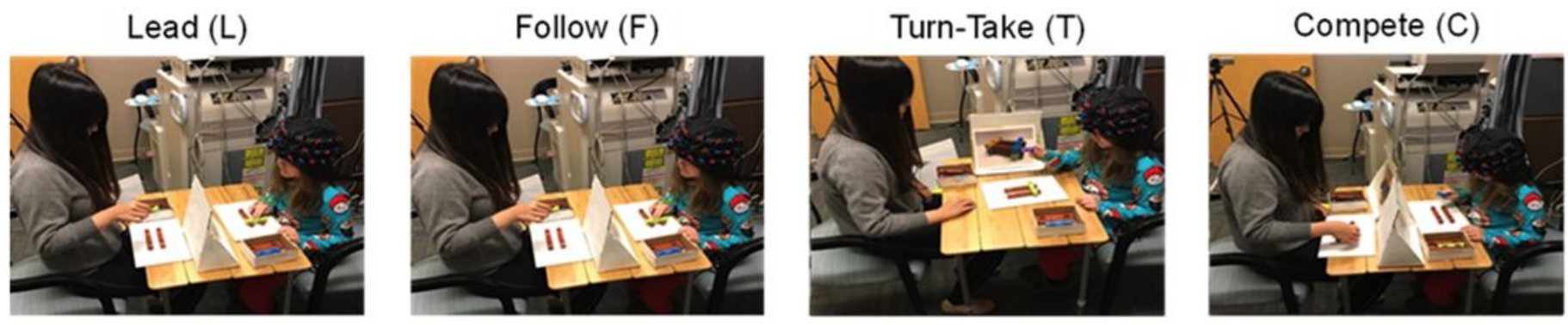

Cooperate (L \& F \& T)

vs

Compete

Synchrony (L \& F)

Turn-Take

Lead

vs

Follow

B. Trial sequence in E-Prime

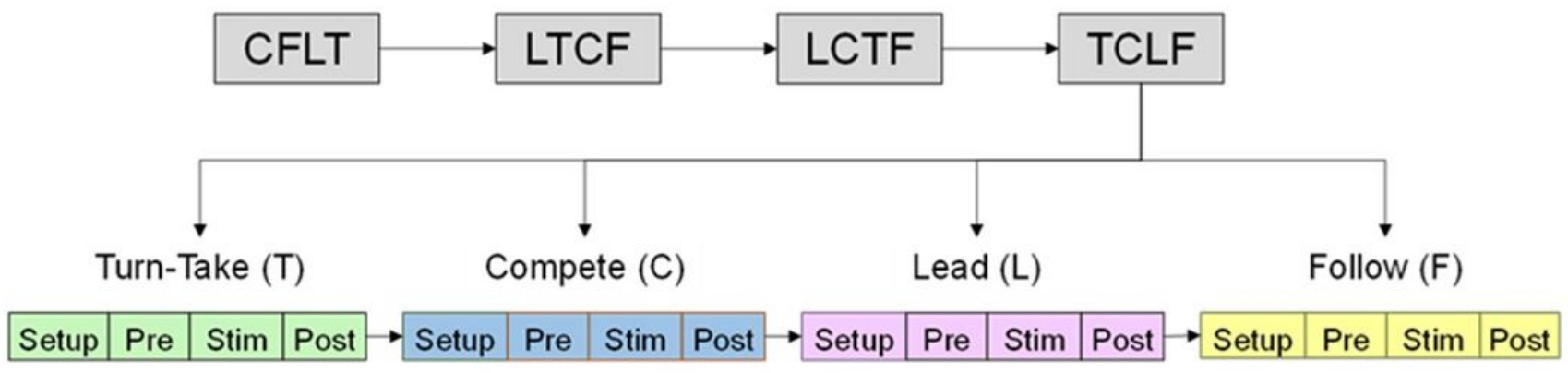

\section{Figure 6}

Experimental conditions of Lead, Follow, Turn-take, and Compete (A), and the randomized blocked design protocol for the joint action task (B).

\section{Supplementary Files}

This is a list of supplementary files associated with this preprint. Click to download.

- 2SupplementaryMaterialsSAG2021.docx 